\title{
El aprendizaje cooperativo como una estrategia de enseñanza del Idioma de Ingles en las Escuelas públicas del Ecuador
}
Cooperative learning as a strategy to teach English Language in the Public Schools of Ecuador.

\section{Aprendizagem cooperativa como estratégia para ensinar o idioma inglês nas escolas públicas do Equado}

Liliana R. Loor-Salmon ${ }^{\mathrm{I}}$

1loor@utm.edu.ec

Mayra M. Palma-Villavicencio II

mpalma@utm.edu.ec

Letty J. Saltos-Rodríguez III

1saltos@utm.edu.ec

Oscar E. Bolívar-Chávez IV

obolivar@utm.edu.ec

Recibido: 14 de enero de 2018 * Corregido: 05 de marzo de 2018 * Aceptado: 10 de junio de 2018

1. Magister En Docencia e Investigación Educativa, Doctora en Educación, Licenciada En Ciencias De La Educación Especialidad Ingles, Docente del Instituto de Lenguas Modernas Universidad Técnica de Manabí, Manta, Ecuador.

II. Magister en Gerencia Educativa, Licenciada en Ciencias de la Educación. Espec. Inglés, Docente de Instituto de Lenguas Modernas, Docente de la Universidad Técnica de Manabí, Manta, Ecuador.

III. Magister en Enseñanza del idioma Ingles, Licenciada en Ciencias de la Educación Mención Ingles Francés, Tecnólogo en Computación Administrativa, Docente del Instituto de Lenguas Modernas Universidad Técnica de Manabí, Manta, Ecuador.

iv. Master en nuevas Tecnologías Aplicadas a la Educación, Departamento de Artes Facultad de Filosofía, Letras y Ciencias de la Educación, Docente de la Universidad Técnica de Manabí, Manta, Ecuador. 


\title{
Resumen
}

El aprendizaje cooperativo, es una estrategia de gran motivación para lograr el aprendizaje de otro idioma. Bajo esta noción, se presenta este artículo cuyo objetivo es proponer actividades de aprendizaje cooperativo para los maestros que trabajan en las Escuelas Públicas Ecuatorianas en pro de motivar en los estudiantes el aprendizaje del inglés. Para ello, se utilizó un enfoque mixto cualicuantitativo. La población la constituyeron los estudiantes del octavo año de educación básica de las escuelas públicas ecuatorianas de la provincia de Tungurahua y cuya muestra fue de 32 estudiantes y 3 profesores de inglés. El instrumento aplicado fue una encuesta de 10 preguntas, cuyas alternativas de repuestas fueron Sí / No y opción múltiple, el análisis de la información obtenida permitió concluir que los profesores de inglés de las Escuelas Pública Ecuatoriana investigadas no aplicaban actividades de aprendizaje cooperativo en sus clases, preferían el trabajo individual para facilitar la evaluación y hacer comentarios individuales sobre la estructura de la gramática y el uso del vocabulario.

Palabras claves: aprendizaje cooperativo; idioma inglés; educación pública y estudiantes.

\begin{abstract}
Cooperative learning is a highly motivating strategy to achieve the learning of another language. Under this notion, this article is presented whose objective is to propose cooperative learning activities for teachers working in the Ecuadorian Public Schools in order to motivate students to learn English. For this, a mixed qualitative-quantitative approach was used. The population was constituted by the students of the eighth year of basic education of the Ecuadorian public schools of the province of Tungurahua and whose sample was of 32 students and 3 teachers of English. The instrument applied was a survey of 10 questions, whose alternatives of answers were Yes / No and multiple choice, the analysis of the information obtained allowed to conclude that the English teachers of the Ecuadorian Public Schools investigated did not apply cooperative learning activities in their classes They preferred individual work to facilitate evaluation and make individual comments on the structure of grammar and the use of vocabulary.
\end{abstract}

Keywords: cooperative learning; english language; public education and students. 


\section{Resumo}

O aprendizado cooperativo é uma estratégia altamente motivadora para alcançar o aprendizado de outro idioma. Sob essa noção, este artigo é apresentado com o objetivo de propor atividades de aprendizagem cooperativa para professores que trabalham nas escolas públicas equatorianas, a fim de motivar os alunos a aprender inglês. Para isso, foi utilizada uma abordagem mista qualitativaquantitativa. A população foi constituída por alunos do oitavo ano do ensino fundamental em escolas públicas província equatoriana de Tungurahua e cuja amostra foi de 32 estudantes e 3 professores de Inglês. $\mathrm{O}$ instrumento utilizado foi uma pesquisa com 10 perguntas cujas respostas alternativas eram sim / não e de múltipla escolha, a análise das informações obtidas permitiram concluir que professores de inglês das escolas públicas equatorianas investigadas não se aplicam actividades de aprendizagem cooperativa em suas aulas. Eles preferiram o trabalho individual para facilitar a avaliação e fazer comentários individuais sobre a estrutura da gramática e o uso do vocabulário.

Palavras chave: aprendizagem cooperativa; idioma inglês; educação pública e estudantes.

\section{Introducción}

El Aprendizaje Cooperativo de Idiomas podría minimizar parte del trabajo de calificación, ya que las lecciones grupales orales no suelen calificarse formalmente. Aunque hay muchos ejemplos de técnicas de aprendizaje cooperativo, los profesores de inglés no están familiarizados con ellos o descubren que las actividades grupales son difíciles de controlar. Por lo general, estos profesores de inglés aplican los métodos tradicionales de enseñanza tales como traducción gramatical, simulacros y repeticiones, repeticiones de vocabulario, actividades individuales en clase, tareas, exámenes estandarizados, etc. Creo que esas actividades no motivan a los alumnos, por lo tanto, ni profesores ni alumnos logran sus objetivos de aprendizaje. Inglés.

Sin embargo, cabe destacar que durante las prácticas docentes en la escuela pública ecuatoriana de la provincia de Tungurahua, a menudo se incluyen actividades de aprendizaje cooperativo. Por ejemplo, diálogos y presentaciones grupales de proyectos, resultando beneficiosos y motivadores para los estudiantes. Por otro lado, se puede observar que la mayoría de los estudiantes no están interesados en el enfoque del Aprendizaje Cooperativo de Idiomas, en tanto que a la mayoría de los estudiantes no les gusta trabajar y realizar actividades de clase en parejas o en grupos, a pesar que están interesados 
por aprender inglés, como puede apreciarse en los 40 alumnos sujetos objetos de esta investigación, en la que la mayoría de ellos prefiere aprender de forma individual. Ello puede estar dándose porque generalmente durante el trabajo en grupo solo los estudiantes más motivados o avanzados trabajan mientras que los demás pierden el tiempo y no hacen su trabajo compartido. Además, en las escuelas públicas ecuatorianas de la ciudad de Tungurahua, hay tres profesores que siempre se quejan de la motivación de los alumnos y la falta de interés por aprender inglés. También se quejan de la cantidad excesiva de tiempo que lleva anotar el trabajo de los estudiantes, como la tarea, los ejercicios de gramática, las actividades del libro de trabajo y la traducción de vocabulario, entre otros. Ante tal planteamiento, se pretende en este artículo proponer actividades de aprendizaje cooperativo para los maestros que trabajan en las Escuelas Públicas Ecuatorianas en alumnos del octavo año de Educación Básica "B" durante el año escolar 2016-2017 en pro de motivar en los estudiantes el aprendizaje del inglés.

\section{Desarrollo}

\section{Aprendizaje cooperativo de idiomas (CLL)}

Según Halili (2013), CLL es parte de un enfoque de instrucción más general también conocido como aprendizaje colaborativo (CL). Es un enfoque que aprovecha al máximo las actividades de cooperación que involucran parejas y pequeños grupos de alumnos en el aula. Para Aroca, Guevara Salazar, Hermosa Tovar y Moreno Solano (2009), el aprendizaje cooperativo es una actividad de aprendizaje grupal organizada para que el aprendizaje dependa del intercambio de información socialmente estructurado entre los alumnos en grupos y en el que cada alumno es responsable de su propio aprendizaje y está motivado para aumentar el aprendizaje de los demás. Asimismo, Johnson, Johnson y Smith, (2013), la indican como "los objetivos de aprendizaje de los estudiantes pueden estructurarse para promover esfuerzos cooperativos, competitivos o individualistas"(pag.33). En cada salón de clases, el objetivo de las actividades es lograr los objetivos y se llevan a cabo por encima de un objetivo en la educación. Por ejemplo, una meta de aprendizaje es un estado futuro anticipado de demostrar competencia o dominio en el área temática que se estudia (Johnson, Johnson y Smith, 2013).

Entendiendo que, la estructura del objetivo define las formas en que los estudiantes interactuarán entre sí y con el instructor durante el período de instrucción. Cada estructura de objetivo tiene su lugar 
(Johnson, Johnson y Smith, 2013). La estructura de objetivo más importante, y la que debería usarse la mayor parte del tiempo en situaciones de aprendizaje, es la cooperación.

De acuerdo con (Johnson y Johnson, 1989, 1999, Johnson, Johnson y Smith, 2006) citado por (Johnson, Johnson y Smith, 2013) Cooperación es trabajar juntos para lograr objetivos compartidos. Dentro de las situaciones de cooperación, los individuos buscan resultados que sean beneficiosos para ellos mismos y beneficiosos para todos los demás miembros del grupo. Por lo tanto, el aprendizaje cooperativo es el uso instructivo de grupos pequeños para que los estudiantes trabajen juntos para maximizar su propio aprendizaje y el de los demás. En este sentido, se puede distinguir con el aprendizaje competitivo en el que los estudiantes trabajan unos contra otros para alcanzar una meta académica más alta y los estudiantes individualistas trabajan solos para lograr metas de aprendizaje aisladas de las del resto de los estudiantes que aprenden. Por lo tanto, en el aprendizaje cooperativo, los maestros evalúan los esfuerzos de los estudiantes sobre una base de criterios referenciados, mientras que, en el aprendizaje competitivo e individualista, los maestros califican a los estudiantes en una referencia normativa (Johnson, Johnson y Smith, 2013).

\section{Enseñanza Cooperativa de Aprendizaje y Lenguaje Comunicativo}

Aunque diferentes investigadores definen el aprendizaje cooperativo de diferentes maneras, la definición operativa de aprendizaje cooperativo implica las siguientes características: el aprendizaje cooperativo es un sistema de enseñanza y aprendizaje en el que los estudiantes son agentes activos en el proceso de aprendizaje en lugar de receptores pasivos del producto de cualquier conocimiento cierto. Este sistema aumenta el aprendizaje académico de los estudiantes, así como el crecimiento personal, ya que disminuye la ansiedad de aprendizaje, aumenta la cantidad de contribución del estudiante y el habla del estudiante en el idioma de destino, también construye entorno de aprendizaje de apoyo y menos amenazante, y ayuda a la tasa de aprendizaje retención. (Masoud Azizinezhad, Hashemi, y Darvishi, 2013). Richards y Platt (1992) citados por Masoud Azizinezhad, Hashemi y Darvishi (2013) señalaron que "las actividades de aprendizaje cooperativo a menudo se utilizan en la enseñanza del lenguaje comunicativo"(pag.65). 


\section{Características generales del aprendizaje cooperativo de idiomas}

Hay algunas características especiales en el aprendizaje cooperativo de idiomas, como la elevación de los logros de todos los estudiantes, el aprendizaje cooperativo promueve las relaciones positivas entre los estudiantes a la vez que proporciona una experiencia de desarrollo social, psicológico y cognitivo saludable y reemplaza la competencia con la cooperación. Reemplaza las lecciones dirigidas por el maestro por actividades grupales e interactivas centradas en el alumno, desarrolla estrategias de aprendizaje y comunicación, reduce el estrés del alumno y crea un clima positivo en el aula (Aroca, Guevara Salazar, Hermosa Tovar y Moreno Solano (2009).

\section{Aprendizaje cooperativo relacionado con el enfoque Teoría del aprendizaje}

Como lo citan Aroca, Guevara Salazar, Hermosa Tovar y Moreno Solano (2009), Piaget y Vygotsky afirman que la teoría del enfoque del aprendizaje es el "Papel de la interacción social en el aprendizaje". Esta teoría de enfoque incluye lo siguiente: desarrollo de habilidades de pensamiento crítico enfatizando en la cooperación, no en la competencia, aumento y variación de la formación de un segundo idioma, desarrollo cognitivo y aumento de las habilidades lingüísticas, integración de lenguaje con áreas basadas en contenido, más variedad de materiales para estimular aprendizaje de lenguaje y conceptos, dominio de habilidades profesionales que resaltan la comunicación y los estudiantes actúan como recursos para el otro en un rol más activo Aroca, Guevara Salazar, Hermosa Tovar y Moreno Solano (2009).

\section{Teoría del lenguaje}

De acuerdo con algunas investigaciones, la capacidad de aprender un lenguaje natural distingue a los humanos de otros animales, y por lo general participa durante la primera década de la vida durante el período crítico para la adquisición del lenguaje. El sistema lingüístico que se desarrolla se infiltra en la vida cotidiana, proporcionando una capacidad lingüística infinita y la creatividad vital del lenguaje. El uso del lenguaje es sensible a una diversidad de variables sociales y contextuales y puede analizarse en varios niveles de descripción. Los lingüistas y otros científicos cognitivos tienen un interés central en la comprensión de la relación entre los idiomas (Halili (2013) y Weeks (1979: 1) citado por Halili (2013), afirma que Coperative Language Learning se basa en algunas premisas básicas sobre la naturaleza interactiva y cooperativa del aprendizaje del lenguaje y el lenguaje "todos los niños 
normales que crecen en un entorno normal aprenden a hablar. Nacemos para hablar. . . Podemos pensar que hemos sido programados para hablar. . . En general, se considera que la comunicación es el propósito principal del lenguaje ". Como lo citan Halili (2013) y según Richards y Schmidt (1983, pag.117)". Los seres humanos pasan gran parte de sus vidas conversando y para la mayoría de ellos, la conversación es una de sus actividades más significativas y apasionantes ".

\section{Teoría del aprendizaje}

Como lo menciona Halili (2013), declarado por Jean Piaget y Lev Vygotsky, "el aprendizaje cooperativo aboga por el trabajo teórico de los psicólogos del desarrollo". Un principio central de CLL es que los alumnos desarrollen competencia comunicativa en un idioma conversando en situaciones social o educativamente estructuradas. CLL también persigue las habilidades de pensamiento crítico de los estudiantes de desarrollo.

\section{Estrategias de aprendizaje cooperativo para ELL}

A los educadores siempre les ha preocupado que los modelos tradicionales de aprendizaje en el aula estén dirigidos por los maestros, fomenten la competencia en lugar de la cooperación, y que los estudiantes de mayoría sean preferidos. Creían que los estudiantes de las minorías podrían estar detrás de los estudiantes de mayor rendimiento. Por lo tanto, hay muchas estrategias de aprendizaje para aplicar en el proceso de enseñanza para involucrar a los estudiantes y motivarlos a aprender inglés. El aprendizaje cooperativo ha demostrado ser efectivo para todo tipo de estudiantes, ya que promueve el aprendizaje y promueve el respeto y las amistades entre diversos grupos de estudiantes. De hecho, cuanto mayor sea la diversidad en un grupo, mayores serán los beneficios para cada estudiante (Halili 2013).

Al aplicar el aprendizaje cooperativo, los compañeros aprenden a depender el uno del otro de manera positiva para una variedad de tareas de aprendizaje. Sin embargo, es necesario que los maestros establezcan normas y procedimientos para el aula que guíen a los estudiantes a contribuir, mantenerse enfocados en la tarea, ayudarse mutuamente, animarse, compartir, resolver problemas, ofrecer y aceptar comentarios de sus compañeros, etc. (Halili 2013). Además, el aprendizaje cooperativo es beneficioso para cualquier estudiante que esté aprendiendo un segundo idioma. Al aplicar actividades de cooperación en el aula de aprendizaje de idiomas, es importante considerar algunas pautas tales 
como: promover la adquisición del lenguaje porque fomenta la interacción entre pares, lo que ayuda a mejorar el lenguaje y el aprendizaje de conceptos y contenido y grupos iguales porque es importante asignar estudiantes ELL a grupos de habilidades mixtas y antecedentes socioeconómicos porque se benefician de los diversos modelos de roles en inglés que componen el grupo (Norman 2013).

Los ELL aprenden a expresarse con mayor confianza en sí mismos cuando trabajan en equipos pequeños. Los ELL se benefician al percibir cómo sus compañeros aprenden y resuelven problemas y "captan" vocabulario de otros en el grupo. Por ejemplo, si los profesores asignan a cada alumno de un equipo un rol como reportero, grabador, cronometrador y administrador de materiales, querrán rotar los roles a medida que la actividad cambie. Al hacer esto, los maestros pueden evitar el problema de que los estudiantes seleccionen los mismos roles. En otras palabras, los mismos estudiantes terminan realizando las mismas tareas, pero si el maestro maneja el juego de roles asignando diferentes roles a los estudiantes, los estudiantes tienen la oportunidad de desarrollar las habilidades que más necesitan para practicar (Norman 2013).

Según Norman (2013), muchas estrategias de aprendizaje cooperativo funcionan con éxito en grupos de cuatro. Esto puede variar de acuerdo con el tamaño y la estructura de la clase. También puede ser útil usar estrategias cooperativas por parejas en lugar de grupos para beneficiar tanto al maestro como a los estudiantes.

\section{Algunos ejemplos de actividades de aprendizaje cooperativo}

Jigsaw es una estrategia de aprendizaje cooperativo que divide a los estudiantes de ELL en grupos, con cada grupo que contiene miembros de varios niveles y habilidades. Los miembros de cada grupo que están en el mismo nivel; trabajen juntos de forma independiente para completar parte de la tarea del grupo. Cuando terminan de trabajar por separado, los miembros del equipo vuelven a sus grupos y trabajan juntos para unir todas sus piezas en un solo proyecto de grupo organizado. En otra actividad, el maestro asignará a cada alumno un número. El profesor plantea una pregunta sobre una lección que ha sido enseñado. Todos los miembros de los grupos deben asegurarse de que cada persona en el grupo sepa cómo responder la pregunta. Luego los maestros llaman un número al azar, y la persona en un grupo al que se llama con ese número debe responder la pregunta para todo el grupo. Por lo tanto, esta actividad ayuda a los estudiantes a aprender unos de otros y promueve la interacción entre iguales. (http://www.colorincolorado.org/article/cooperative-learning-strategies) 
Escribir alrededor es otro ejemplo de una actividad de aprendizaje cooperativo. En Write Around, el maestro comienza dividiendo la clase en grupos de cuatro o más según el tamaño de la clase. Luego él / ella les da una historia o resumen inicial una oración larga que cada persona en cada grupo puede agregar una oración. Luego, cada grupo puede presentar sus historias o resúmenes de una clase a la clase. (http://www.colorincolorado.org/article/cooperative-learning-strategies)

Tea Party es una actividad en la que los estudiantes se alinean en dos líneas una frente a la otra. El docente plantea una pregunta a la clase y pide a los alumnos que se enfrenten en las líneas que discutan la pregunta durante un minuto o dos según el tamaño del grupo. Luego, el docente plantea una nueva pregunta y pide que una línea se desplace hacia la derecha para que cada alumno se encuentre frente a una nueva pareja. Estos nuevos socios discuten la pregunta y así sucesivamente. Esta actividad también se puede hacer en círculos, donde un círculo se mueve hacia la derecha cuando es el momento de conseguir nuevos socios (http://www.colorincolorado.org/article/cooperative-learning-strategies).

\section{Papel del docente en el aprendizaje cooperativo}

De acuerdo con Johnson et al. (1998 a, b, 2002) citado por Gillies, Ashman y Terwel (2008), el aprendizaje cooperativo es el uso instructivo de grupos pequeños para que los estudiantes trabajen juntos para maximizar su propio aprendizaje y el de los demás. Existen tres tipos de aprendizaje cooperativo, como grupos de base formales, informales y cooperativos.

\section{Aprendizaje cooperativo Formal}

El Aprendizaje Cooperativo Formal, consiste en que los estudiantes trabajen juntos durante un período de clase a varias semanas para lograr objetivos de aprendizaje compartidos y completar tareas y asignaciones conjuntamente específicas de acuerdo con (Johnson et al., 1998a, b, 2002). Como lo citan Gillies, Ashman y Terwel (2008) en el libro "El papel del docente en la implementación del aprendizaje cooperativo en el aula" en grupos formales de aprendizaje cooperativo, el papel de los docentes es el siguiente: formular objetivos de habilidades académicas y sociales, decidir sobre el tamaño de los grupos, elija un método para asignar estudiantes a los grupos, decida qué roles asignar a los miembros del grupo, organice la sala y coloque los materiales que los alumnos necesitan para 
completar la tarea. $\mathrm{Al}$ asignar roles a los estudiantes, se establece la interdependencia de roles. (Gillies, Ashman, y Terwel, 2008).

La forma en que se distribuyen los materiales crea interdependencia de recursos. La disposición de la sala crea interdependencia ambiental y proporciona al docente un acceso fácil para observar a cada grupo, lo que aumenta la responsabilidad individual y proporciona datos para el procesamiento grupal. Al explicar la tarea de instrucción y la estructura cooperativa, los maestros aclaran la tarea académica a los estudiantes, explican los criterios de rendimiento, proporcionan una estructura que fomenta la interdependencia y la responsabilidad discreta, explican los comportamientos que se espera que usen los estudiantes y enfatizan la cooperación intergrupal. De esta forma, los docentes eliminan la posibilidad de competencia entre los estudiantes y extienden la interdependencia de objetivos positivos a la clase (Gillies, Ashman y Terwel, 2008).

Además, al explicar las habilidades sociales resaltadas en la lección, los profesores controlan lo siguiente: los objetivos de habilidades sociales de la lección y los patrones de interacción, monitoreando el aprendizaje de los estudiantes e interviniendo para proporcionar asistencia para completar la tarea de manera efectiva utilizando las habilidades interpersonales y grupales eficazmente. Mientras dirigen la lección, los maestros monitorean cada grupo de aprendizaje y los asisten cuando es necesario para mejorar el trabajo de la tarea y el trabajo en equipo.

El monitoreo de los grupos de aprendizaje crea responsabilidad individual. Cada vez que los maestros observan a un grupo, los miembros tienden a sentirse responsables de ser miembros constructivos. Además, los maestros recopilan datos específicos para promover la interacción, el uso de habilidades sociales específicas y el compromiso con los patrones de interacción deseados (Gillies, Ashman y Terwel, 2008).

Mientras evalúan el aprendizaje de los estudiantes y ayudan a los estudiantes a definir qué tan bien funcionan sus grupos, cierran la lección, evalúan y evalúan la calidad y cantidad del rendimiento estudiantil, aseguran que los estudiantes analicen cuidadosamente la eficacia con la que trabajaron juntos, ayudan a los estudiantes a elaborar un plan de mejora; y alentar a los estudiantes a celebrar el arduo trabajo de los miembros del grupo. La evaluación del rendimiento del alumno destaca la responsabilidad individual y grupal e indica si el grupo logró sus objetivos. 
La celebración del grupo es una forma de recompensar la interdependencia. La retroalimentación recibida durante el procesamiento grupal está dirigida a mejorar el uso de las habilidades sociales y es una forma de responsabilidad individual. Al hablar de los procesos, el grupo solía funcionar, enfatiza la mejora continua y promueve la interacción y los patrones de interacción necesarios para maximizar el aprendizaje y la retención de los estudiantes (Gillies, Ashman y Terwel, 2008). De acuerdo con Johnson et al. (1998b, 2002) citado por Gillies, Ashman y Terwel, (2008).

\section{Aprendizaje Cooperativo informal}

El mismo consiste en hacer que los estudiantes trabajen juntos para lograr objetivos de aprendizaje mutuo en grupos temporales que duran unos minutos a un período de clase durante una conferencia o demostración. El aprendizaje cooperativo informal se puede utilizar para centrar la atención del alumno en el material que se debe aprender, ayudar a los alumnos a establecer expectativas, garantizar que los alumnos procesen y ensayen cognitivamente el material que se enseña. El aprendizaje cooperativo informal permite a los maestros resumir lo que se aprendió y cerrar la sesión de instrucción. Los roles del docente en el uso del aprendizaje cooperativo informal son los siguientes: hacer la tarea y las instrucciones claras y precisas, y exigir que los grupos produzcan un producto específico, como una respuesta escrita (Gillies, Ashman y Terwel, 2008). El procedimiento de roles de los maestros se presenta de la siguiente manera:

Discusión introductoria enfocada en la cual los maestros asignan a los estudiantes a explicar la tarea de responder las preguntas en un período de tiempo de 4 o 5 minutos y la interdependencia positiva de llegar a un consenso. La tarea de discusión tiene como objetivo promover la organización anticipada de lo que los estudiantes saben sobre el tema a ser presentado y establecer expectativas sobre lo que la conferencia cubrirá. La responsabilidad individual está garantizada por el pequeño tamaño del grupo. Se requiere un patrón básico de interacción para estimular la práctica oral, el pensamiento de alto nivel y la construcción de acuerdos (Gillies, Ashman y Terwel, 2008). Discusiones enfocadas intermitentes en las cuales los maestros dividen la conferencia en partes de 10 o 15 minutos. Después de cada sección, se les pide a los estudiantes que se dirijan a la persona que se encuentra junto a ellos y trabajen cooperativamente respondiendo preguntas que requieran que los estudiantes procesen cognitivamente el material presentado. 
La técnica procede de la siguiente manera: cada alumno formula su respuesta, los estudiantes comparten su respuesta con su compañero, los alumnos escuchan atentamente la respuesta de su compañero y los pares construyen una nueva respuesta que es más alta para la pregunta inicial de cada miembro. La pregunta puede requerir que los estudiantes resuman con el material presentado, reaccionen a los conceptos y pronostiquen qué se presentará a continuación, resuelvan un problema, compartan material con el aprendizaje anterior e íntegro en un marco conceptual. Los docentes deben asegurarse de que los estudiantes persigan el consenso para llegar a un acuerdo sobre las respuestas a las preguntas (Gillies, Ashman y Terwel, 2008). Los profesores aleatoriamente eligen dos o tres estudiantes para presentar un resumen de 30 segundos de sus discusiones. Tal responsabilidad individual asegura que las parejas tomen

las tareas en serio y se controlan entre sí para asegurarse de que ambos estén preparados para responder. Periódicamente, el maestro debe estructurar la interdependencia social. Discusión enfocada en el cierre en la cual los maestros les dan a los estudiantes una tarea final de discusión que dura de 4 a 5 min. La tarea requiere que los estudiantes resuman lo que han aprendido de la conferencia e integren en un marco conceptual existente. La tarea también puede apuntar a los alumnos hacia lo que se presentará en la próxima sesión de clase. Esto proporciona el cierre de la conferencia. El aprendizaje cooperativo informal asegura que los estudiantes participen con entusiasmo en la comprensión de lo que se está presentando. También proporciona tiempo para que los maestros caminen alrededor de la clase escuchando lo que dicen los estudiantes. Al escuchar las discusiones de los estudiantes, los instructores les dan dirección y entendimiento sobre cuán bien los estudiantes aprecian los conceptos y el material, así como también aumentan la responsabilidad individual de participar en las discusiones (Gillies, Ashman y Terwel, 2008).

\section{Aprendizaje cooperativo}

El último tipo de aprendizaje cooperativo es el de Grupos de base cooperativos. De acuerdo con Johnson et al. 1998b, (2002) citado por Gillies, Ashman y Terwel, (2008), "Los grupos de base cooperativos son grupos de aprendizaje cooperativo heterogéneos a largo plazo con una membresía estable". Las principales responsabilidades de los miembros son las siguientes: Certificar que todos los miembros están logrando un buen progreso educativo, se hacen responsables mutuamente y se motivan para aprender y brindarse apoyo, aliento y ayuda mutua para completar las tareas. 
Periódicamente, los maestros deben enseñar las habilidades sociales requeridas y hacer que el proceso grupal funcione eficazmente para garantizar que los grupos base funcionen de manera efectiva. Los grupos de base cooperativos generalmente son heterogéneos en cuanto a membresía en términos de logros, motivación y orientación de tareas. La agenda del grupo base puede incluir tareas de apoyo académico, por ejemplo, conocerse entre sí y ayudarse mutuamente a resolver problemas no académicos, tareas rutinarias y tareas de evaluación y verificar la comprensión mutua. Por lo tanto, la función del docente en el uso de grupos de base cooperativos es formar grupos heterogéneos y programar un horario en el que se reúnen regularmente al comienzo y al final de cada clase (Gillies, Ashman y Terwel, 2008).

Mientras más tiempo exista un grupo cooperativo, entre más cariñosas sean sus relaciones, mayor será el apoyo social que se brindarán entre sí, más comprometidos estarán con el éxito del otro y más influencia tendrán los miembros sobre los demás. (Gillies, Ashman, y Terwel, 2008). Ventajas y desventajas del aprendizaje cooperativo, Aunque el sentido más básico del aprendizaje cooperativo es el trabajo en grupo en el que dos o más estudiantes trabajan juntos para completar una tarea de clase, el aprendizaje cooperativo presenta algunas desventajas. Los siguientes son considerados desventajas: Los mismos grados; Esfuerzo desigual Al calificar un grupo, todos los miembros de un grupo reciben el mismo grado sin importar cuánto trabajo haga cada miembro. Esto es una desventaja para los miembros del grupo que hacen la mayoría del trabajo. Además, cuando los proyectos como los informes escritos se clasifican, puede ser difícil para el profesor concluir qué partes del proyecto completaron cada miembro del grupo. Para combatir la posible desigualdad, el docente puede monitorear cuidadosamente a los grupos mientras trabajan para definir las contribuciones de cada miembro (Sosnowski, 2001). Los grupos pueden ser demasiado sociales Los grupos cooperativos pueden ser creados por la selección de estudiantes o profesores, y cada uno tiene algunas desventajas. Cuando los estudiantes pueden seleccionar sus propios grupos, pueden elegir basándose únicamente en las preferencias sociales, lo que puede alentar a los alumnos a perder de vista la tarea asignada. Por otro lado, si el docente selecciona grupos, existe el peligro de agruparse estudiantes de habilidades similares, que pueden crear grupos de estudiantes muy débiles. También existe la posibilidad de agrupar a los estudiantes que pueden no trabajar bien juntos (Sosnowski, 2001).

La planificación de la lección puede llevar más tiempo Cuando los estudiantes trabajan en grupos, la tarea del maestro es administrar toda el aula, en este sentido es un poco más difícil ya que se requiere 
que los estudiantes interactúen. El maestro puede tener que diseñar una lección y una evaluación con anticipación que sea apropiada para el trabajo grupal y que permita la evaluación imparcial de todos los estudiantes. El maestro también puede necesitar desarrollar una tarea que involucre a todos los miembros del grupo durante el tiempo asignado. Además, los maestros deben tener cuidado con el plagio en el trabajo grupal, particularmente si se requiere que cada miembro presente su propio proyecto final. (Sosnowski, 2001). Haciéndolo una buena experiencia de aprendizaje para todos. El aprendizaje cooperativo exitoso ofrece una experiencia positiva de comunicación y trabajo en equipo. Para construir estas oportunidades, una planificación cuidadosa puede mejorar algunas de las desventajas encontradas en el trabajo grupal. En calificar la injusticia se puede evitar generando expectativas claras para cada miembro del grupo. La agrupación informal, como contar números grupales, puede disminuir la cantidad de conversaciones innecesarias y mantener a los estudiantes concentrados en sus tareas. Finalmente, los maestros pueden planificar actividades de aprendizaje cooperativo para revisar las lecciones y habilidades ya aprendidas, por lo tanto, el trabajo en grupo no será la única forma en que los estudiantes aprenden sobre un tema. (Sosnowski, 2001).

\section{Actividades de clase para el aprendizaje cooperativo}

Según Devlin (2001), "el aprendizaje cooperativo es un estilo de educación que hace que los estudiantes participen activamente en el aula". El aprendizaje cooperativo es mucho más que trabajo grupal, aunque eso es parte de él. La Universidad Estatal de Kennesaw define el aprendizaje cooperativo como cuando "los equipos pequeños ... usan una variedad de actividades de aprendizaje para mejorar su comprensión de un tema". Este método de educación es apropiado para todos los niveles de edad y para todas las asignaturas, algunas actividades de la clase incluyen lo siguiente:

\section{Rompecabezas}

El método Jigsaw agrupa a los estudiantes, generalmente en grupos de cinco. Cada alumno aprende un tema diferente y luego se lo enseña a los demás miembros del grupo. El maestro luego hace preguntas y probablemente realiza un examen. Por ejemplo, a un alumno se le puede pedir que estudie y explique sobre el ascenso de Hitler al poder, mientras que el otro puede centrarse en la entrada y el papel de Japón en la guerra (Devlin 2001). 
Actividades para llegar a conocerlo Preguntas y actividades para romper el hielo son importantes para fomentar el trabajo en equipo y la cooperación. Una forma de hacerlo es encontrar una pelota de playa grande y escribir preguntas por todas partes, luego los maestros hacen que los estudiantes se paren en un círculo grande, ya sea en el aula o afuera, para que se lancen la pelota entre ellos. Quien atrape la pelota tiene que responder la pregunta que el pulgar derecho del alumno está o está más cerca. Por ejemplo, las preguntas de ejemplo incluyen "¿Cuál es tu película favorita?", "¿Cuál es tu materia favorita en la escuela?" y "¿Por qué eligió el atuendo que lleva puesto hoy?", etc. (Devlin 2001). Diario de doble entrada El Diario de doble entrada es la mejor actividad que se realiza con los estudiantes en parejas al trabajar juntos en un solo proyecto. Cada estudiante revisa el desarrollo y las contribuciones del otro en el lado izquierdo de una hoja de papel. Una vez que se completa el proyecto, los estudiantes intercambian papeles para que tengan las observaciones del otro. Después de un día de considerar los comentarios, los estudiantes pueden escribir sus respuestas en el lado derecho del documento. Para fomentar aún más la cooperación, las parejas deben reunirse por última vez para analizar cualquier inquietud y sugerencia, así como para buscar las aclaraciones que necesiten (Devlin 2001).

\section{Mesa redonda}

En un ejercicio de mesa redonda, los estudiantes pueden sentarse en un círculo. Luego, el docente da un tema y cada alumno, dando vueltas en el círculo, tiene que ofrecer una palabra o idea en un cierto límite de tiempo. Para los estudiantes principiantes, el tema puede ser tan simple como pedirles que propongan palabras que comiencen con cierta letra. Por ejemplo, se les puede pedir a los estudiantes competentes que aporten ideas sobre cómo evaluar el tema o dar sugerencias sobre el tema presentado (Devlin 2001).

\section{Escribir alrededor}

Esta actividad es similar al método de la mesa redonda. El maestro puede poner un comienzo de historia en la parte superior de una hoja de papel, y luego comenzar a pasar la hoja alrededor del salón de clases. Cada alumno agranda una oración propia. Una vez que la hoja se ha pasado al menos una vez, el maestro puede leer su historia en voz alta. Esto también puede funcionar para estudiantes mayores y sujetos fuera del inglés. Por ejemplo, un profesor de historia o de ciencias puede preguntar a los alumnos sobre cada hecho presente sobre un tema específico. (Devlin 2001). 
Concluyendo, sobre la base de la investigación, este documento propone actividades cooperativas de crianza como actividades interactivas para los estudiantes que están aprendiendo un segundo idioma, ya que les da a los estudiantes la oportunidad de compartir su conocimiento de acuerdo con sus antecedentes. Al aplicar el aprendizaje cooperativo, los maestros pueden administrar grandes aulas y al mismo tiempo los maestros pueden monitorear fácilmente la colaboración de los estudiantes en el grupo. Mientras los estudiantes trabajan en grupos, los maestros pueden observar el aprendizaje de los estudiantes y motivarlos para que aprendan. Por lo tanto, los maestros tienen tiempo para enseñar un contenido innovador en lugar de perder el tiempo en calificar el trabajo individual de los estudiantes. Basado en el análisis, recomiendo tres actividades de aprendizaje cooperativo. Considero que estas actividades son beneficiosas para los estudiantes de octavo grado de Educación Básica en la Escuela Mariano Benítez. Por ejemplo, Rompecabezas, Actividades para llegar a conocer y mesa redonda. Este tipo de actividades motiva e involucra a los alumnos para aprender. Cuando los docentes aplican Jigsaw, Actividades para Conocer y Mesa Redonda, les permiten a los estudiantes no solo aprender, sino también enseñar entre ellos de acuerdo con sus antecedentes socioculturales y el nivel de conocimiento de los estudiantes dependiendo del tema.

\section{Metodología}

La investigación siguió un enfoque mixto cuali-cuantitativo. En cuanto a lo cualitativo, a partir de la proposición de materiales y actividades para enseñar un segundo idioma, de acuerdo a Hidalgo (2017) y Leedy y Ormrod (2001). En cuanto a la metodología Cuantitativa permitió proporcionar un análisis descriptivo y frecuencial de los datos aportados por los investigados, tal como se presentaron en su contexto o estado específico, en la que se identificaron las características del fenómeno Creswell (2003) citado por (Hidalgo (2017). En cuanto al método de estudio, se refirió a un estudio de caso que permitió identificar un caso o situación específica y buscar posibles soluciones a un problema específico, permitiendo proporcionar un método para analizar el proceso de enseñanza y aprendizaje, el problema de investigación y proponer algunas soluciones posibles basadas en la recopilación de datos de encuestas y entrevistas, a tal efecto Yin (1984: 23) citado por Zainal (2007), define el método de investigación del estudio de caso "como una investigación empírica que investiga un fenómeno contemporáneo dentro de su contexto de la vida real; cuando los límites entre el fenómeno y el contexto no son claramente evidentes; y en el que se usan múltiples fuentes de evidencia "(pag.77). La población la constituyeron los estudiantes del octavo año de educación básica de las escuelas 
Dom. Cien., ISSN: 2477-8818

Vol. 4, núm. 3, julio, 2018, pp. 431-448

El aprendizaje cooperativo como una estrategia de enseñanza del Idioma de Ingles en las Escuelas públicas del

LOMS CIENCIAS Ecuador

públicas ecuatorianas de la provincia de Tungurahua y cuya muestra fue de 32 estudiantes y 3 profesores de inglés. El instrumento aplicado fue una encuesta de 10 preguntas, cuyas alternativas de repuestas fueron Sí / No y opción múltiple.

\section{Análisis de los Resultados}

La categorización fue utilizada para analizar las encuestas. Con base en el análisis de las encuestas, quedó claro que los profesores de inglés de la Escuela Pública Ecuatoriana no aplicaban actividades de aprendizaje cooperativo en sus clases. Prefieren que los estudiantes trabajen individualmente porque de esta forma los maestros pueden evaluar y hacer comentarios individuales sobre la estructura de la gramática y el uso del vocabulario. Según la encuesta, también está claro que a algunos de los estudiantes no les gusta trabajar en grupos porque no todos los miembros del grupo comparten sus ideas correctamente o participan con el mismo esfuerzo. Sin embargo, un número moderado de estudiantes prefieren practicar aprendizaje cooperativo porque se sienten más libres de aportar su propio conocimiento sobre un tema determinado.

\section{Conclusiones}

Se pudo constatar que existe entre los alumnos investigados desconocimiento acerca del beneficio del aprendizaje cooperativo durante el aprendizaje del idioma de Ingles.

Se hace necesario, que los alumnos y docentes investigados comprendan las ventajas de la cooperación, en el entendido que con el aprendizaje cooperativo se tiene la oportunidad no solo de aprender sino también de enseñarse mutuamente.

Un alto porcentaje de los alumnos investigados, rechazan el trabajo en grupos lo cual fue evidenciado al responder aquellas preguntas que requerían la estrategia de cooperación de sus compañeros.

Se pudo constatar, que los docentes participantes estaban motivados en aplicar actividades de aprendizaje cooperativo en el aula durante su proceso de enseñanza, específicamente debido a la cantidad de alumnos en cada aula, sería mejor y más beneficioso pedirles a los participantes que trabajen de forma cooperativa, permitiendo reducir la pérdida de tiempo de los docentes al tener que puntuar tantas actividades individuales. 
Dom. Cien., ISSN: $2477-8818$

Vol. 4, núm. 3, julio, 2018, pp. 431-448

El aprendizaje cooperativo como una estrategia de enseñanza del Idioma de Ingles en las Escuelas públicas del

LAS CIENCIAS Ecuador

\section{Referencias Bibliográficas}

Aroca, W. B., Guevara Salazar, Y. E., Hermosa Tovar, J. A., \& Moreno Solano, J. L. (2009, November 24). Educación, Tecnología. Retrieved from https://es.slideshare.net/guest7flad678/cooperativelanguage-learning

Darvishi (2013). Application of Cooperative Learning. Recuperado en: https://www.researchgate.net/.../275537643_Application_of_Coo.

Devlin, N. (2001). Leaf Group Ltd English Language. Retrieved from http://classroom.synonym.com/classroom-activities-cooperative-learning-7916223.html

Gillies, R. M., Ashman, A., \& Terwel, J. (2008). Social Interdependence Theory and Cooperative Learning: The Teacher's Role. New York: Springer Science+Business Media LLC, 233 Spring Street New York.

Halili, D. J. (2013, April 15). Education. Retrieved from https://es.slideshare.net/DenLynHalili/cooperative-language-learning-18854783

Hidalgo (2017). Panorama. Recuperado en: https://www.researchgate.net/.../Panoramas-de-laenseñanza-de-las-lenguas-extranjeras.

Johnson, D. W., Johnson, R. T., \& Smith, K. A. (2013). Cooperative Learning: Improving University Instruction by Basing Practice On Validated Theory. Journal on Excellence in University Teaching, 26.

Masoud Azizinezhad, Hashemi, M., \& Darvishi, S. (2013). Application of cooperative learning in EFL classes to enhance the the Students' Language Learning. Procedia - Social and Behavioral Sciences, 138-141.

Norman, L. (2013, 12 31). Bright Hub Education. Retrieved from http://www.brighthubeducation.com/esl-teaching-tips/99116-using-cooperative-learning-in-youresl-class/

Sosnowski, J. (2001). Leaf Group Ltd. English Language. Retrieved from $\mathrm{http} / / /$ classroom.synonym.com/three-disadvantages-using-cooperative-learning-10044274.html (http://www.colorincolorado.org/article/cooperative-learning-strategies).

https://www.smartsheet.com/15-free-task-list-templates http://www.dictionary.com/browse/teaching? s=t 